Ibn Al-Haitham Jour. for Pure \& Appl. Sci. 33 (2) 2020

Ibn Al Haitham Journal for Pure and Applied Science

Journal homepage: http://jih.uobaghdad.edu.iq/index.php/j/index

\title{
Alternating Directions Implicit Method for Solving Homogeneous Heat Diffusion Equation
}

\section{Bushra Sh. Mahmood}

Department of Mathematic /College of Computer Science and Mathematics /Tikrit University/

Tikrit/Iraq.

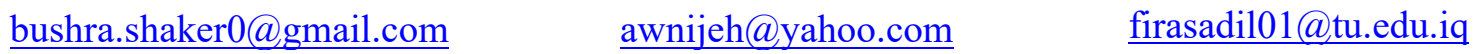

Firas A. Fawzi

Article history: Received 7 July 2019, Accepted 8 September 2019, Published in April 2020.

Doi: $10.30526 / 33.2 .2427$

\section{Abstract}

An Alternating Directions Implicit method is presented to solve the homogeneous heat diffusion equation when the governing equation is a bi-harmonic equation $(\mathrm{X})$ based on Alternative Direction Implicit (ADI). Numerical results are compared with other results obtained by other numerical (explicit and implicit) methods. We apply these methods it two examples $(\mathrm{X})$ : the first one, we apply explicit when the temperature $\geq 0$.

Keywords: Alternating Directions Implicit (ADI) method, Diffusion Heat Equation, BiHarmonic equation.

\section{Introduction}

$\mathrm{A} \mathrm{Bi}-\mathrm{Harmonic}$ equation is a kind of partial differential equations, the general form of the bi- harmonic equation is:

$\nabla^{4} u(x, y)=F(x, y)$

Where $\nabla^{4}$ is a bi -harmonic operator in the form:

$\nabla^{4}=\frac{\partial^{4}}{\partial x^{4}}+2 \frac{\partial^{4}}{\partial x^{2} \partial y^{2}}+\frac{\partial^{4}}{\partial y^{4}}$

If $\nabla^{4} u(x, y)=0$, then it is called homogenous bi- harmonic function.

This equation appears in many boundary value problems: (fluid mechanics, elasticity problems, heat diffusion, etc...), when the governing equation for the boundary value problems is a bi harmonic equation. There are many $(\mathrm{X})$ numerical methods to solve this problem. One of these methods is Alternating Direct Implicit Method (ADI). This method is to minimize the two dimensions for a partial differential equation to a linear equation with one dimension. The Bi-harmonic equation is the governing for many $(\mathrm{X})$ problems, for example: it is the governing for the heat equation. Several researchers presented the heat 
equation, see [1-4]. Moreover, Turck [5]. (X) solve the heat equation. Then, Poulilikkas et. [6]. Constructed methods of fundamental solution for harmonic and Biharmonic BVPs. Martin and Ismael [7]. (X) proposed TH-collection for biharmnic equation. Subsequently, Jacob [8]. (X) presented the (a) comparative study of (a) 2D asymmetric diffusion problem With convection on the wall using the theta method. Furthermore, Noye and Hayman in [9]. utilized ADI to solve the 2D time dependent heat equations depending on a constant coefficient. Donough in [10]. (X) used ADI methods for solving elliptic problems. Aderito [11]. Hyperbolic diffusion equation with convection used an alternating direction implicit method for a second-order. The main purpose of this work is to study the analytic and numerical solution for solving homogeneous heat diffusion equation using ADI method.

\section{Alternating Directions Implicit Method}

This method is one technique of finite different methods. There are many methods of (ADI), for examples: Peaceman -Rachford, Douglas - Gunn, and Fairweather-Mitchel method and all these methods have absolutely stable with respect the heat equations. The basic idea for (ADI) method is summarized by the following steps:

Step1: apply the implicit method in X-direction and the explicit method in Y-direction.

Step2: Solve the equation in time $\left(n+\frac{1}{2}\right)$.

Step3: Apply the implicit method in Y-direction and the explicit method in X-direction, as follows:

$$
\begin{aligned}
& \frac{\boldsymbol{T}_{i j}^{n+\frac{1}{2}}-\boldsymbol{T}_{i j}^{n}}{\Delta t / 2}=\left(\delta_{x}^{2} \boldsymbol{T}_{i j}^{n+\frac{1}{2}}+\delta_{y}^{2} \boldsymbol{T}_{i j}^{n}\right)^{2} \\
& \frac{\boldsymbol{T}_{i j}^{n+1}-\boldsymbol{T}_{i j}^{n+\frac{1}{2}}}{\Delta t / 2}=\left(\delta_{x}^{2} \boldsymbol{T}_{i j}^{n+\frac{1}{2}}+\delta_{y}^{2} \boldsymbol{T}_{i j}^{n+1}\right)^{2}
\end{aligned}
$$

Where $\delta_{x}^{2}$ and $\delta_{y}^{2}$ are the second derivatives with respect to $\mathrm{x}$ and $\mathrm{y}$, sequentially and the general form for the diffusion heat equation is [2].

$V_{i j}^{n+1}=\left(1+r \delta_{x}^{4}+\frac{r}{8} \delta_{x y}^{2}+r \delta_{y}^{4}\right) V_{i j}^{n}$, where $\mathrm{r}=\frac{k}{h^{4}}$, and $\mathrm{h}=\Delta x=\Delta y, \mathrm{k}$ is

Step4: Solve this equation by using tridiagonal matrix algorithm (Thomas algorithm).

\section{Solve Bi-harmonic homogenous heat diffusion equation by ADI method}

The governing heat diffusion $\mathrm{Bi}$ - harmonic equation is

$$
\nabla^{4} T=\frac{\partial^{4} T}{\partial x^{4}}+2 \frac{\partial^{4} T}{\partial x^{2} \partial y^{2}}+\frac{\partial^{4} T}{\partial y^{4}}
$$

With the boundary conditions :(Dirichlet boundary condition, Neumann boundary condition, and Robin boundary condition).

Now, we explain how to solve bi- harmonic equation by one of (ADI) methods (Peaceman Rachford method):

First, we take the following Biharmonic equation:

$$
u_{x x x x}+2 u_{x x y y}+u_{y y y y}
$$

And we exchange the values of the function in equation (6) with its finite differences, which is: 
Ibn Al-Haitham Jour. for Pure \& Appl. Sci. 33 (2) 2020

$$
\begin{aligned}
& u_{x x x x}=\frac{u_{i+2, j}-4 u_{i+1, j}+6 u_{i j}-4 u_{i-1, j}+u_{i-2, j}}{h^{4}} \\
& u_{y y y y}=\frac{u_{i, j+2}-4 u_{i, j+1}+6 u_{i j}-4 u_{i, j-1}+u_{i, j-2}}{h^{4}} \\
& 2 u_{x x y y}=\frac{u_{i+2, j+2}+u_{i-2, j-2}+4 u_{i, j}+u_{i+2, j-2}+u_{i-2, j+2}-2 u_{i, j+2}-2 u_{i+2, j}-2 u_{i-2, j}-2 u_{i, j-2}}{8 h^{4}}
\end{aligned}
$$

\section{Practical Part}

In this section we apply the ADI method to solve two heat diffusion problems.

\section{Example 1}

If we have a rectangular metal plate which is 4 inch wide and 8 inch high are applicable to the $\mathrm{x}$ and $\mathrm{y}$ axes, at the point of origin according to the data shown in the figure below. $\mathrm{T}_{\mathrm{i}}^{\mathrm{m}+1}=\mathrm{T}_{\mathrm{i}}^{\mathrm{m}}+\lambda\left(\mathrm{T}_{\mathrm{i}-1}^{\mathrm{m}}-2 \mathrm{~T}_{\mathrm{i}}^{\mathrm{m}}+\mathrm{T}_{\mathrm{i}+1}^{\mathrm{m}}\right)$

Where, $\lambda=\frac{k \Delta t}{(\Delta t)^{2}}, \Delta t$ is the variation in time, $\Delta x$ is the variation in $\mathrm{x}$ direct, $\mathrm{k}$ is thermal conductivity coefficient, and $\mathrm{m}$ is the levels.

$$
\begin{aligned}
& T_{1}^{1}=T_{1}^{0}+\lambda\left(T_{0}^{0}-2 T_{1}^{0}+T_{1}^{0}\right)=0+0.2(4-2(0)+0)=0.8 \\
& T_{2}^{1}=T_{2}^{0}+\lambda\left(T_{1}^{0}-2 T_{2}^{0}+T_{3}^{0}\right)=0+0.2(0-2(0)+0)=0 \\
& T_{3}^{1}=T_{3}^{0}+\lambda\left(T_{2}^{0}-2 T_{3}^{0}+T_{4}^{0}\right)=0+0.2(0-2(0)+2)=0.4
\end{aligned}
$$

\begin{tabular}{l|l|l|l|l|l|l|}
$t=6$ & Left & & & & & Right \\
$t=4$ & boundary & & & & boundary \\
$t=2$ & $4^{\circ} \mathrm{C}$ & & & & & \\
\cline { 2 - 5 }$t=0$ & & $0.8^{\circ} \mathrm{C}$ & $0^{\circ} \mathrm{C}$ & $0.4^{\circ} \mathrm{C}$ & & \\
\hline
\end{tabular}

Figure 1. The nodes in the first level.

$t=2, m=1$ to find $m=2, t=4, m$ is the levels

$T_{1}^{2}=T_{1}^{1}+\lambda\left(T_{0}^{1}-2 T_{1}^{1}+T_{2}^{1}\right)=0.8+0.2(4-2(0.8)+0)=1.28$

$T_{2}^{2}=T_{2}^{1}+\lambda\left(T_{1}^{1}-2 T_{2}^{1}+T_{3}^{1}\right)=0+0.2(0.8-2(0)+0.4)=0.24$

$T_{3}^{2}=T_{3}^{1}+\lambda\left(T_{2}^{1}-2 T_{3}^{1}+T_{4}^{1}\right)=0.4+0.2(0-2(0.4)+2)=0.96$

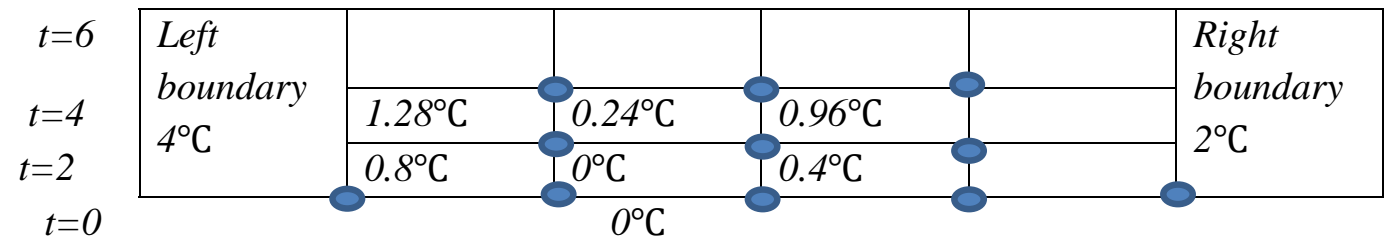

Figure 2. The nodes in the second level. 
Ibn Al-Haitham Jour. for Pure \& Appl. Sci. 33 (2) 2020

$$
\begin{aligned}
& t=2, m=1 \text { to find } m=3, t=6 \\
& T_{1}^{3}=T_{1}^{2}+\lambda\left(T_{0}^{2}-2 T_{1}^{2}+T_{2}^{2}\right)=1.28+0.2(4-2(1.28)+0.24)=1.616 \\
& T_{2}^{3}=T_{2}^{2}+\lambda\left(T_{1}^{2}-2 T_{2}^{2}+T_{3}^{2}\right)=0.24+0.2(1.28-2(0.24)+0.96)=0.592 \\
& T_{3}^{3}=T_{3}^{2}+\lambda\left(T_{2}^{2}-2 T_{3}^{2}+T_{4}^{2}\right)=0.96+0.2(0.24-2(0.96)+2)=1.024
\end{aligned}
$$

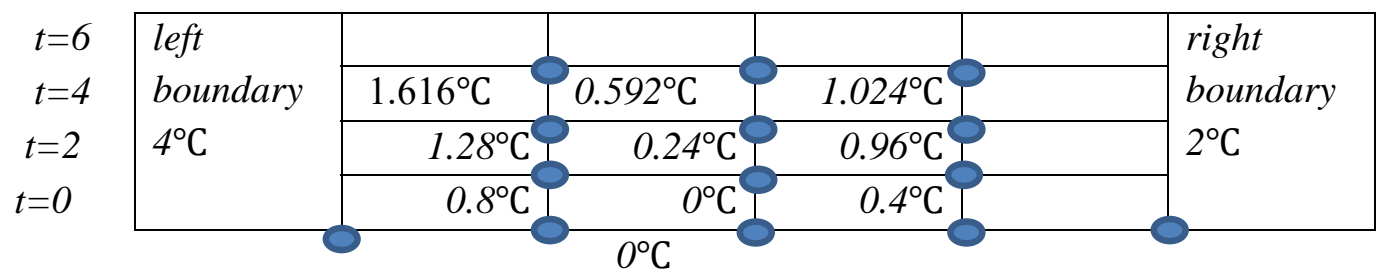

Figure 3. The nodes in the third level.

By Implicit Method

If $m=0$ to find $m=1, \lambda=0.2$

At the left boundary $\quad(1+2 \lambda) T_{1}^{1}-\lambda T_{2}^{1}=T_{1}^{0}+\lambda T_{0}^{1}$

$(1+2(0.2)) T_{1}^{1}-0.2 T_{2}^{1}=0+0.2(4)$

Away from boundary $-\lambda T_{i-1}^{1}+(1+2 \lambda) T_{i}^{1}-\lambda T_{i+1}^{1}=T_{1}^{0}$

$-0.2 T_{1}^{1}+(1+2(0.2)) T_{2}^{1}-0.2 T_{3}^{1}=0$

At the Right boundary $(1+2 \lambda) T_{i}^{m+1}-\lambda T_{i-1}^{m+1}=T_{i}^{m}+\lambda T_{i+1}^{m+1}$

$(1+2(0.2)) T_{3}^{1}-0.2 T_{2}^{1}=T_{3}^{0}+0.2 T_{4}^{1}$

We have

$$
\begin{aligned}
& 1.4 T_{1}^{1}-0.2 T_{2}^{1}=0.8 \\
& -0.2 T_{1}^{1}+1.4 T_{2}^{1}-0.2 T_{3}^{1}=0 \\
& 1.4 T_{3}^{1}-0.2 T_{2}^{1}=0+0.4
\end{aligned}
$$

We have

$T_{1}^{1}=\frac{0.8+0.2 T_{2}^{1}}{1.4}, T_{3}^{1}=\frac{0.4+0.2 T_{2}^{1}}{1.4}$

And by compensation, we get

$-0.2\left(\frac{0.8+0.2 T_{2}^{1}}{1.4}\right)+1.4 T_{2}^{1}-0.2\left(\frac{0.4+0.2 T_{2}^{1}}{1.4}\right)=0$

$-0.1142857143-0.0285714286 T_{2}^{1}+1.4 T_{2}^{1}-0.0571428571-0.0285714286 T_{2}^{1}=0$

$1.3428571428 T_{2}^{1}=0.174285714$

$T_{2}^{1}=0.1276595745$

And compensation $T_{2}^{1}$ in $T_{1}^{1}$ and $T_{3}^{1}$ we get $T_{3}^{1}=0.3039513678, T_{1}^{1}=0.5896656535$

If $m=1$ to find $m=2$

At the left boundary $(1+2 \lambda) T_{1}^{2}-\lambda T_{2}^{2}=T_{1}^{1}+\lambda T_{0}^{2}$

$(1+2(0.2)) T_{1}^{2}-0.2 T_{2}^{2}=0.5896656535+0.2(4)$ 
Away from boundary $-\lambda T_{1}^{2}+(1+2 \lambda) T_{2}^{2}-\lambda T_{3}^{2}=T_{2}^{1}$

$-0.2 T_{1}^{2}+(1+2(0.2)) T_{2}^{2}-0.2 T_{3}^{2}=0.1276595745$

At the Right boundary $(1+2 \lambda) T_{i}^{m+1}-\lambda T_{i-1}^{m+1}=T_{i}^{m}+\lambda T_{i+1}^{m+1}$

$(1+2(0.2)) T_{3}^{2}-0.2 T_{2}^{2}=T_{3}^{1}+0.2 T_{4}^{2}$

We have

$1.4 T_{1}^{2}-0.2 T_{2}^{2}=1.3896656535$

$-0.2 T_{1}^{2}+1.4 T_{2}^{2}-0.2 T_{3}^{2}=0.1276595745$

$1.4 T_{3}^{2}-0.2 T_{2}^{2}=0.7039513678$

So, we have, $T_{1}^{2}=\frac{1.3896656535+0.2 T_{2}^{2}}{1.4}, T_{3}^{2}=\frac{0.7039513678+0.2 T_{2}^{2}}{1.4}$.

$-0.2\left(\frac{1.3896656535+0.2 T_{2}^{2}}{1.4}\right)+1.4 T_{2}^{2}-0.2\left(\frac{0.7039513678+0.2 T_{2}^{2}}{1.4}\right)=0.1276595745$

$-0.1985236648-0.0285714286 T_{2}^{2}+1.4 T_{2}^{2}-0.1005644811-0.0285714286 T_{2}^{2}=$

0.1276595745

$1.3428571428 T_{2}^{2}=0.4267477204$

$T_{2}^{2}=0.3177908556$

And compensation $T_{2}^{2}$ in $T_{1}^{2}$ and $T_{3}^{2}$ we get

$T_{3}^{2}=0.5482210992, T_{1}^{2}=1.0380170176$

If $m=2$ to find $m=3$

At the left boundary $\quad(1+2 \lambda) T_{1}^{1}-\lambda T_{2}^{3}=T_{1}^{2}+\lambda T_{0}^{3}$

$(1+2(0.2)) T_{1}^{3}-0.2 T_{2}^{3}=1.0380170176+0.2(4)$

Away from boundary $-\lambda T_{i-1}^{3}+(1+2 \lambda) T_{i}^{3}-\lambda T_{i+1}^{3}=T_{i}^{2}$

$-0.2 T_{1}^{3}+(1+2(0.2)) T_{2}^{3}-0.2 T_{3}^{3}=0.3177908556$

At the Right boundary $(1+2 \lambda) T_{i}^{m+1}-\lambda T_{i-1}^{m+1}=T_{i}^{m}+\lambda T_{i+1}^{m+1}$

$(1+2(0.2)) T_{3}^{3}-0.2 T_{2}^{3}=T_{3}^{2}+0.2 T_{4}^{3}$

$1.4 T_{3}^{3}-0.2 T_{2}^{3}=0.5482210992+0.2(2)$

So, we have

$1.4 T_{1}^{3}-0.2 T_{2}^{3}=1.8380170176$

$-0.2 T_{1}^{3}+1.4 T_{2}^{3}-0.2 T_{3}^{3}=0.3177908556$

$1.4 T_{3}^{3}-0.2 T_{2}^{3}=0.948221099$

So, we have

$T_{1}^{3}=\frac{1.8380170176+0.2 T_{2}^{3}}{1.4}$

$T_{3}^{3}=\frac{0.9482210992+0.2 T_{2}^{3}}{1.4}$

And by compensation, we get

$-0.2\left(\frac{1.8380170176+0.2 T_{2}^{3}}{1.4}\right)+1.4 T_{2}^{3}-0.2\left(\frac{0.9482210992+0.2 T_{2}^{3}}{1.4}\right)=0.3177908556$

$-0.2625738597-0.0285714286 T_{2}^{3}+1.4 T_{2}^{3}-0.135460157-0.0285714286 T_{2}^{3}=$

0.3177908556

$1.3428571428 T_{2}^{3}=0.7158248723$ 
Ibn Al-Haitham Jour. for Pure \& Appl. Sci. 33 (2) 2020

$T_{2}^{3}=0.5330610751$

And compensation $T_{2}^{3}$ in $T_{1}^{3}$ and $T_{3}^{3}$ we get

$T_{3}^{3}=0.7534523673, T_{1}^{3}=1.3890208804$

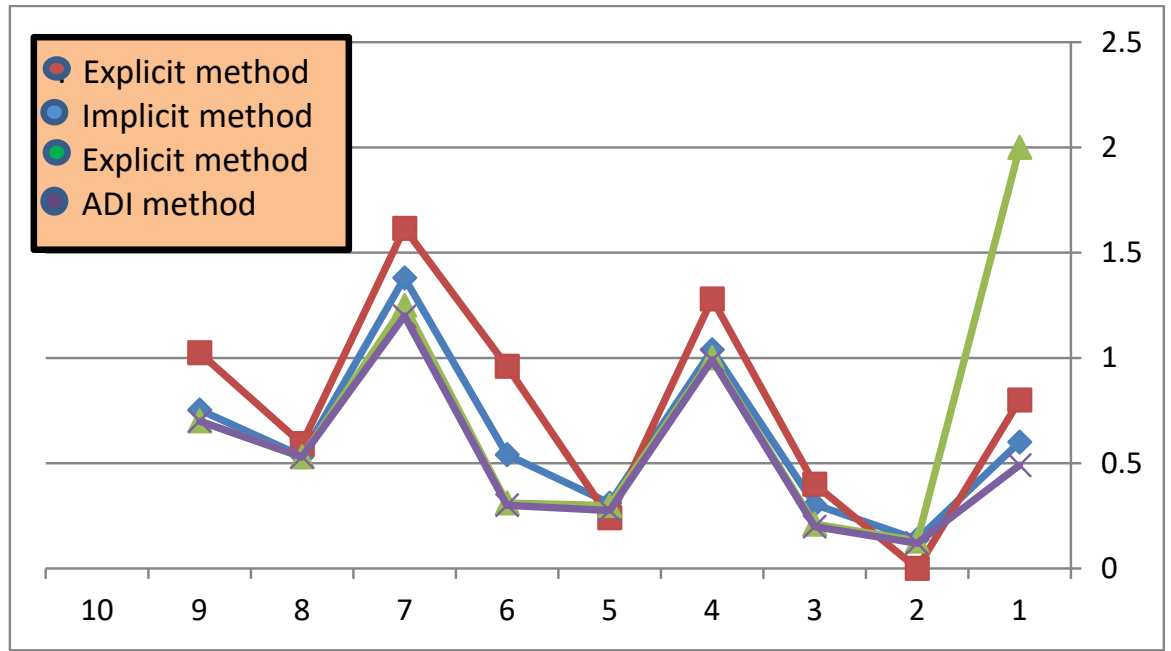

Figure 4. Comparing between ADI, explicit, implicit, exact solution when the temperature $\geq 0$.

From the previous figures, we see the ADI method is most accuracy of the implicit and explicit method.

\section{Example 2}

We have an important material that needs to be stored in a cupboard whose temperature does not exceed (5) degrees. It is necessary to check the efficiency of the insulating material made up of the cabinet in the case of power failure for different periods, which affects the storage process according to the following data.

$T_{1}^{1}=T_{1}^{0}+\lambda\left(T_{0}^{0}-2 T_{1}^{0}+T_{1}^{0}\right)=0+0.2(-2-2(0)+0)=-0.4$
$T_{2}^{1}=T_{2}^{0}+\lambda\left(T_{1}^{0}-2 T_{2}^{0}+T_{3}^{0}\right)=0+0.2(0-2(0)+0)=0$
$T_{3}^{1}=T_{3}^{0}+\lambda\left(T_{2}^{0}-2 T_{3}^{0}+T_{4}^{0}\right)=0+0.2(0-2(0)+(-4))=-0.8$

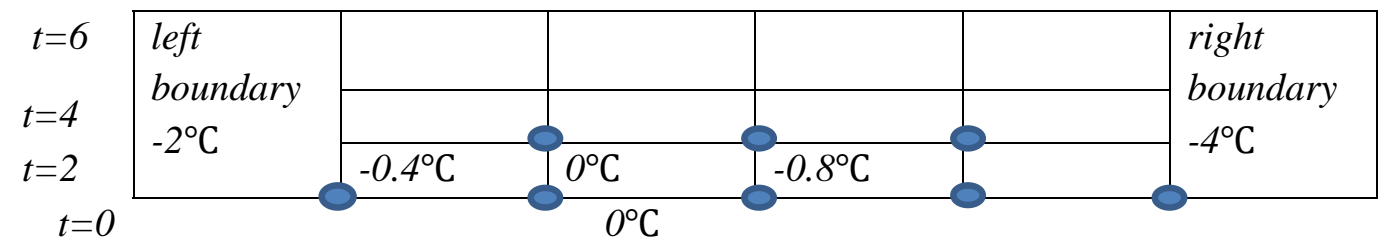

Figure 5.The nodes in the first level when temperature $\leq 0$.

$$
\begin{aligned}
& T_{1}^{2}=T_{1}^{1}+\lambda\left(T_{0}^{1}-2 T_{1}^{1}+T_{2}^{1}\right)=-0.4+0.2(-2-2(-0.4)+0)=-0.64 \\
& T_{2}^{2}=T_{2}^{1}+\lambda\left(T_{1}^{1}-2 T_{2}^{1}+T_{3}^{1}\right)=0+0.2(-0.4-2(0)+(-0.8))=-0.24 \\
& T_{3}^{2}=T_{3}^{1}+\lambda\left(T_{2}^{1}-2 T_{3}^{1}+T_{4}^{1}\right)=-0.8+0.2(0-2(-0.8)+(-4))=-1.28
\end{aligned}
$$


Ibn Al-Haitham Jour. for Pure \& Appl. Sci. 33 (2) 2020

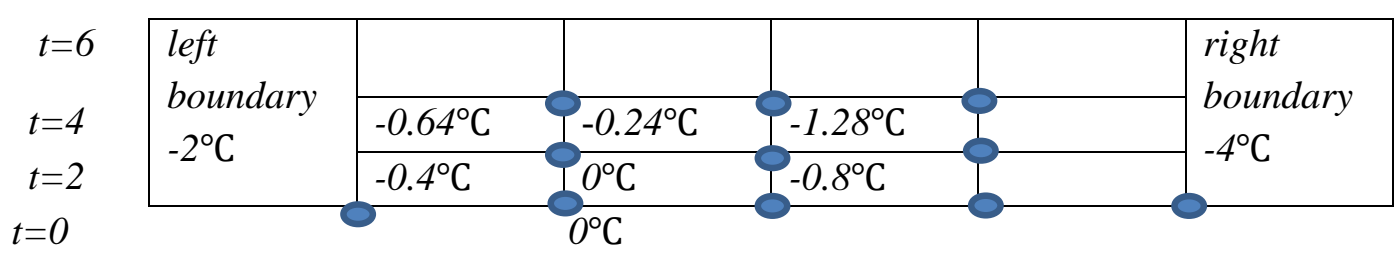

Figure 6.The nodes in the second level when temperature $\leq 0$.

$$
\begin{aligned}
& T_{1}^{3}=T_{1}^{2}+\lambda\left(T_{0}^{2}-2 T_{1}^{2}+T_{2}^{2}\right)=-0.64+0.2(-2-2(-0.64)+(-0.24))=-0.528 \\
& T_{2}^{3}=T_{2}^{2}+\lambda\left(T_{1}^{2}-2 T_{2}^{2}+T_{3}^{2}\right)=-0.24+0.2(-0.64-2(-0.24)+(-1.28))=-1.216 \\
& T_{3}^{3}=T_{3}^{2}+\lambda\left(T_{2}^{2}-2 T_{3}^{2}+T_{4}^{2}\right)=-1.28+0.2(-0.24-2(-1.28)+(-2))=-1.216
\end{aligned}
$$

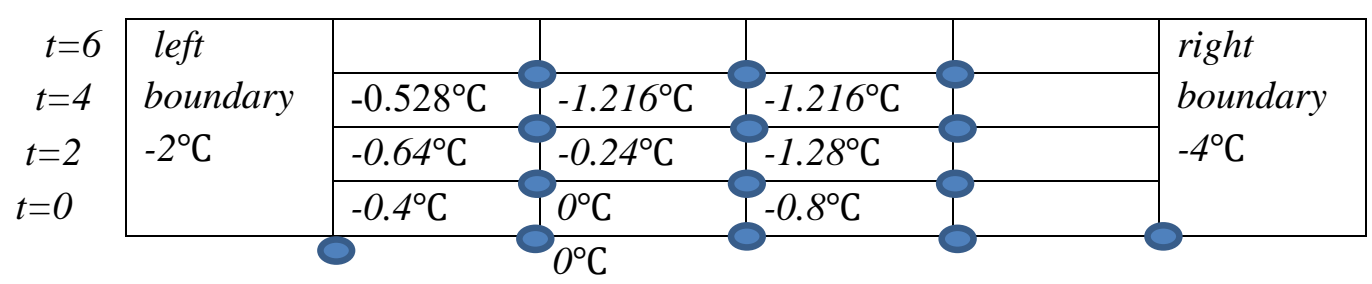

Figure 7. The nodes in the third level when temperature $\leq 0$.

By implicit method

At the left boundary $\quad(1+2 \lambda) T_{1}^{m+1}-\lambda T_{2}^{m+1}=T_{1}^{m}+\lambda T_{0}^{m+1}$

Away from boundary $\quad-\lambda T_{i-1}^{m+1}+(1+2 \lambda) T_{i}^{m}-\lambda T_{i+1}^{m+1}=T_{1}^{m}$

At the Right boundary $(1+2 \lambda) T_{i}^{m+1}-\lambda T_{i-1}^{m+1}=T_{i}^{m}+\lambda T_{i+1}^{m+1}$

If $m=0$ to find $m=1$

$(1+2(0.2)) T_{1}^{1}-0.2 T_{2}^{1}=T_{1}^{0}+0.2 T_{0}^{1}$

$-0.2 T_{1}^{1}+(1+2(0.2)) T_{2}^{1}-0.2 T_{3}^{1}=T_{2}^{0}$

$(1+2(0.2)) T_{3}^{1}-0.2 T_{2}^{1}=T_{3}^{0}+0.2 T_{4}^{1}$

$1.4 T_{1}^{1}-0.2 T_{2}^{1}=0+0.2(-2)$

$-0.2 T_{1}^{1}+1.4 T_{2}^{1}-0.2 T_{3}^{1}=0$

$1.4 T_{3}^{1}-0.2 T_{2}^{1}=0+0.2(-4)$

We have

$1.4 T_{1}^{1}-0.2 T_{2}^{1}=-0.4$

$-0.2 T_{1}^{1}+1.4 T_{2}^{1}-0.2 T_{3}^{1}=0$

$1.4 T_{3}^{1}-0.2 T_{2}^{1}=-0.8$

Implies that

$T_{1}^{1}=\frac{-0.4+0.2 T_{2}^{1}}{1.4}$ 
$T_{3}^{1}=\frac{-0.8+0.2 T_{2}^{1}}{1.4}$

And the compensation, we get

$-0.2\left(\frac{-0.4+0.2 T_{2}^{1}}{1.4}\right)+1.4 T_{2}^{1}-0.2\left(\frac{-0.8+0.2 T_{2}^{1}}{1.4}\right)=0$

$0.0571428571-0.0285714286 T_{2}^{1}+1.4 T_{2}^{1}+0.1142857143-0.0285714286 T_{2}^{1}=0$

$1.3428571428 T_{2}^{1}=-0.1714285714$ implies that $T_{2}^{1}=-0.1276595745$

And compensation in $T_{1}^{1}$ and $T_{3}^{1}$ we get, $T_{1}^{1}=-0.3039513678$

$$
T_{3}^{1}=-0.5896656535
$$

If $m=1$ to find $m=2$

$$
\begin{aligned}
& (1+2(0.2)) T_{1}^{2}-0.2 T_{2}^{2}=T_{1}^{1}+0.2 T_{0}^{2} \\
& -0.2 T_{1}^{2}+(1+2(0.2)) T_{2}^{2}-0.2 T_{3}^{2}=T_{2}^{1} \\
& (1+2(0.2)) T_{3}^{2}-0.2 T_{2}^{2}=T_{3}^{1}+0.2 T_{4}^{2}
\end{aligned}
$$

$1.4 T_{1}^{2}-0.2 T_{2}^{2}=-0.3039513678+0.2(-2)$

$-0.2 T_{1}^{2}+1.4 T_{2}^{2}-0.2 T_{3}^{2}=-0.1276595745$

$1.4 T_{3}^{2}-0.2 T_{2}^{2}=-0.5896656535+0.2(-4)$

We have

$$
\begin{aligned}
& 1.4 T_{1}^{2}-0.2 T_{2}^{2}=-0.7039513678 \\
& -0.2 T_{1}^{2}+1.4 T_{2}^{2}-0.2 T_{3}^{2}=-0.1276595745 \\
& 1.4 T_{3}^{2}-0.2 T_{2}^{2}=-0.3896656535
\end{aligned}
$$

Hence,

$T_{1}^{2}=\frac{-0.7039513678+0.2 T_{2}^{2}}{1.4}$

$T_{3}^{2}=\frac{-1.3896656535+0.2 T_{2}^{2}}{1.4}$

And by compensation we get,

$-0.2\left(\frac{-7039513678+0.2 T_{2}^{2}}{1.4}\right)+1.4 T_{2}^{2}-0.2\left(\frac{-1.3896656535+0.2 T_{2}^{2}}{1.4}\right)=-0.1276595745$

$0.1005644811-0.0285714285 T_{2}^{2}+1.4 T_{2}^{2}+0.1985236648-0.0285714286 T_{2}^{2}=-0.1276595745$ $1.3428571428 T_{2}^{1}=-0.4267477204$ implies that $T_{2}^{2}=-0.3177908556$

And compensation in $T_{1}^{2}$ and $T_{3}^{2}$, we get $T_{1}^{2}=-0.5482210992, T_{3}^{2}=-1.0380170176$

If $m=2$ to find $m=3$

$$
\begin{aligned}
& (1+2(0.2)) T_{1}^{3}-0.2 T_{2}^{3}=T_{1}^{2}+0.2 T_{0}^{3} \\
& -0.2 T_{1}^{3}+(1+2(0.2)) T_{2}^{3}-0.2 T_{3}^{3}=T_{2}^{2} \\
& (1+2(0.2)) T_{3}^{3}-0.2 T_{2}^{3}=T_{3}^{2}+0.2 T_{4}^{3} \\
& 1.4 T_{1}^{3}-0.2 T_{2}^{3}=-0.5482210992+0.2(-2) \\
& -0.2 T_{1}^{3}+1.4 T_{2}^{3}-0.2 T_{3}^{3}=-0.3177908556
\end{aligned}
$$




$$
1.4 T_{3}^{3}-0.2 T_{2}^{3}=-1.0380170176+0.2(-4)
$$

We have

$1.4 T_{1}^{3}-0.2 T_{2}^{3}=-0.9482210992$

$-0.2 T_{1}^{3}+1.4 T_{2}^{3}-0.2 T_{3}^{3}=-0.3177908556$

$1.4 T_{3}^{3}-0.2 T_{2}^{3}=-1.8380170176$

Which is implies that

$T_{1}^{3}=\frac{-0.948221099+0.2 T_{2}^{3}}{1.4}, T_{3}^{3}=\frac{-1.8380170176+0.2 T_{2}^{3}}{1.4}$

So, we get

$-0.2\left(\frac{-0.948221099+0.2 T_{2}^{3}}{1.4}\right)+1.4 T_{2}^{3}-0.2\left(\frac{-1.8380170176+0.2 T_{2}^{3}}{1.4}\right)=-0.3177908556$

$0.135460157-0.0285714285 T_{2}^{3}+1.4 T_{2}^{3}+0.2625738597-0.0285714286 T_{2}^{3}=-0.3177908556$

$1.3428571428 T_{2}^{3}=-0.7158248723$ implies that $T_{2}^{3}=-0.5330610751$

And compensation in $T_{1}^{2}$ and $T_{3}^{2}$ we get, $T_{1}^{3}=-0.7534523672, T_{3}^{2}=-1.3890208804$

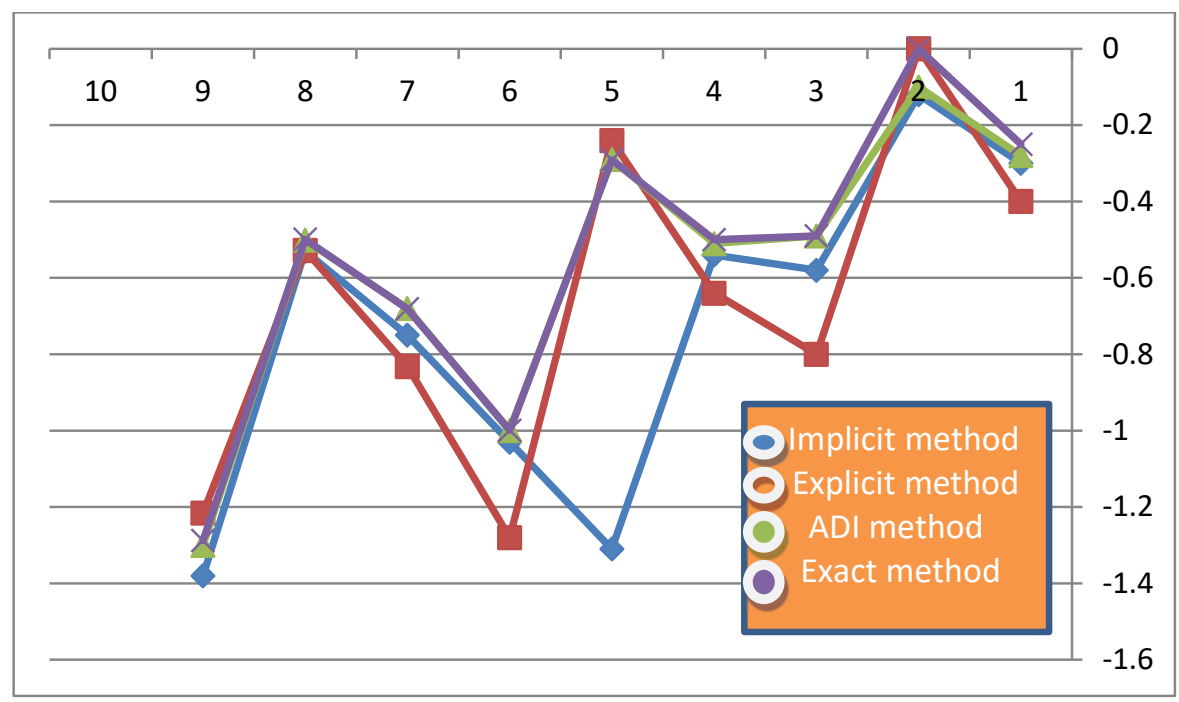

Figure 8. Comparison between ADI, implicit, explicit, exact solution when the temperature $\leq 0$.

According to the previous figures, we see the ADI method is most accuracy of the implicit and explicit.

\section{Conclusion}

In this paper, the Alternating Direction Implicit (ADI) method has been constructed for the purpose of application to some selected problems with a view to discuss the accuracy of this technique, as shown in figures (4 and 8) when compared with exact solution. Then, many problems have been solved. Figures illustrate the numerical results of the new method are more efficient and desirable for the solution of the homogeneous heat diffusion equation than other methods. 
Ibn Al-Haitham Jour. for Pure \& Appl. Sci. 33 (2) 2020

\section{References}

1. Widder, D.V. The Heat Equation, $1^{\text {st }}$ ed., Academic press, New York, San Francisco, London, 1975, ISBN: 9780080873831.

2. Matthew, J.H. The 1-D Heat Equation.2006, Available from: https://pdfs.semanticscholar.org/0e31/9ff7d1f0833e6f084255fcd89f06167f00c4.pdf

3. Horak, V.; Gruber, P. Parallel Numerical Solution of 2-D Heat Equation, Parallel Numerics.2005, 5, 47-56.

4. Gockenbach, M.; Schmidtke, K. Newton's Law Of Heating And The Heat Equation, Involv. J. Math.2009, 2, 419-437.

5. De-Turck, D. Solving the Heat Equation, University of Pennsylvania, USA, Math.2012, 241, 1-12.

6. Poullikkas, A.; Karageorghis, A.; Georgiou, G. Methods Of Fundamental Solutions For Harmonic And Biharmonic Boundary Value Problems, Comput Mech.1998, 21, 416423.

7. Martin, D.; Ismael, H. TH-Collocation for the Biharmonic Equation, Advances in Engineering Software.2005, 36, 243-251.

8. Jacob, N. Theta Method Comparative Study Of 2D Asymmetric Diffusion Problem With Convection On The Wall, Wseas transactions on fluid mechanics.2015, 10, 35-46.

9. Noye B. J.; Hayman, K.J. New LOD and ADI Methods for The Two Dimensional Diffusion Equation, J. computer Mathematics.1994, 51, 215-228.

10. Peaceman, D.W., Rachford, H.H. The Numerical Solution of Parabolic and Elliptic Differential Equations, J.soc. Indust. Appl. Math.1955, 3, 28-4.

11. Aderito, A.; Cidalia, N.; Ercilia, S. An Alternating Direction Implicit Method For A Second-Order Hyperbolic Diffusion Equation With Convection, Applied Mathematics and Computation.2014, 239, 17-28. 